\title{
A Prototype of a Novel Energy Efficient Variable Stiffness Actuator
}

\author{
L.C. Visser*, R. Carloni*, F. Klijnstra** and S. Stramigioli*
}

\begin{abstract}
In this work, we present a proof of concept of a novel variable stiffness actuator. The actuator design is based on the conceptual design proposed in earlier work, and is such that the apparent output stiffness of the actuator can be changed independently of the output position and without any energy cost. Experimental results show that the behavior of the prototype is in accordance with the theoretical results of the conceptual design, and thus show that energy efficient variable stiffness actuators can be realized.
\end{abstract}

\section{INTRODUCTION}

Humans can perform tasks in many different conditions and environments since they are able to properly adjust the stiffness of their joints. In contrast, robot actuation is usually stiff and is not suitable in applications in which robots need to cooperate with humans, such as in prosthetics, rehabilitation or wearable devices and social robots. Therefore, robots should have capabilities similar to humans, and be capable of adjusting the joint stiffness to the task and environment. This can be achieved by using actuators, of which the apparent output stiffness, and thus the joint stiffness, can be changed.

For this kind of actuators, commonly called variable stiffness actuators, the apparent output stiffness can be changed independently of the output position by means of a number of internal springs and actuated degrees of freedom, which determine how the springs are sensed at the output of the actuator. Based on this concept, a number of variable stiffness actuators have been presented in recent years, including AMASC [1], VSA [2], VS-Joint [3], and MACCEPA [4]. These actuators rely on the pretension of one or more springs, in series with the actuator output, to change the output stiffness. This means that, to change the stiffness, the amount of energy stored in the springs is changed. The consequence is that energy is supplied to the actuator without doing work at the output, i.e. energy is required to change the stiffness.

In a recent work, we presented a general port-based model for variable stiffness actuators [5]. From the analysis of the model, design criteria were derived for energy efficient variable stiffness actuators, and it was shown that it is possible to design actuators in which the stiffness can be changed without using energy. Based on this, we designed a conceptual actuator and validated the design with simulations. In this paper, we present a prototype realization

This work has been funded by the European Commission's Seventh Framework Programme as part of the project VIACTORS under grant no. 231554.

* \{1.c.visser,r.carloni,s.stramigioli $\} @$ utwente.nl, Department of Electrical Engineering, Faculty of Electrical Engineering, Mathematics and Computer Science, University of Twente, 7500 AE Enschede, The Netherlands.

**f.klijnstra@student.utwente.nl, Deptartment of Advanced Technology, Faculty of Science and Technology, University of Twente, 7500 AE Enschede, The Netherlands.

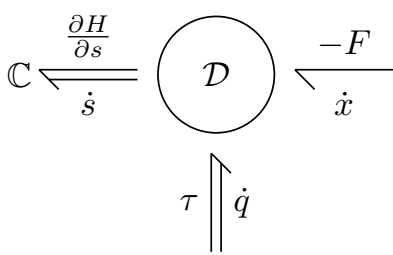

Fig. 1. Generalized representation of a variable stiffness actuator - The Dirac structure $\mathcal{D}$ defines the interconnection between the different elements and, therefore, how power is distributed among the ports. The multi-bonds allow any number of springs, i.e. the $\mathbb{C}$-element, and any number of control inputs $(\tau, \dot{q})$. The one dimensional port $(-F, \dot{x})$ is the output port.

of the conceptual design. We show that experiments are in accordance with the simulation results, and thus we can validate the concept. In particular, we show that the apparent output stiffness of the prototype actuator can be changed in an energy free way, and that energy supplied to the actuator is used only to do work on the load.

\section{PORT-BASED MODEL OF VARIABLE STIFFNESS ACTUATORS}

In this Section, we recall the model of variable stiffness actuators, derived by using the port-based modeling formalism [6] and extensively described in [5]. The portbased framework captures the essential properties of variable stiffness actuators in terms of energy and, in particular, it provides important insights in the power flows between the actuator, the actuated system and the controller of the internal degrees of freedom of the actuator.

In the formulation of the model, we assume that:

- the variable stiffness actuator has internal springs;

- there are actuated degrees of freedom that determine how the springs are sensed at the output of the actuator;

- internal friction and inertias can be neglected.

The model is graphically depicted in Fig. 1 by using bond graphs. The multi-dimensional $\mathbb{C}$-element represents the internal springs of the actuator and is characterized by a state $s$, i.e. the elongation or compression of the springs, and by an energy function $H(s)$, describing the amount of elastic energy stored by the springs. The power conjugate port variables of the element are the flow $\dot{s}$, the rate of change of the internal state, and, dually, the forces exerted by the springs, i.e. the effort $\frac{\partial H}{\partial s}$.

The internal degrees of freedom of the actuator are actuated via the control port $(\tau, \dot{q})$, where the effort variable $\tau$ describes the generalized forces that actuate the degrees of freedom and the flow variable $\dot{q}$ denotes the generalized rate of change of the configuration variables $q$. The output port of the actuator is denoted by $(-F, \dot{x})$, where the effort $F$ is 
the force generated by the actuator and the flow $\dot{x}$ is the rate of change of the output position $x$.

The Dirac structure $\mathcal{D}$ defines how the power is distributed between the bonds. The structure is power continuous, and thus defines a constraint relation between the power variables of the connected bonds. In matrix form, the Dirac structure is represented by means of a skew symmetric matrix $D(q, x)$ :

$$
\left[\begin{array}{c}
\dot{s} \\
\tau \\
-F
\end{array}\right]=\underbrace{\left[\begin{array}{ccc}
0 & A(q, x) & B(q, x) \\
-A(q, x)^{T} & 0 & C(q, x) \\
-B(q, x)^{T} & -C(q, x)^{T} & 0
\end{array}\right]}_{D(q, x)}\left[\begin{array}{c}
-\frac{\partial H}{\partial s} \\
\dot{q} \\
\dot{x}
\end{array}\right]
$$

Since gyration does not regularly exist in the mechanical domain, $C(q, x)=0$. Note that the Dirac structure is not necessarily constant and is allowed to depend on both the configuration of the internal degrees of freedom $q$ and the actuator output position $x$.

The rate of change of the energy stored in the springs is:

$$
\begin{aligned}
\frac{\mathrm{d} H}{\mathrm{~d} t} & =\frac{\partial H}{\partial s} \frac{\mathrm{d} s}{\mathrm{~d} t}=\frac{\partial H}{\partial s}(A(q, x) \dot{q}+B(q, x) \dot{x}) \\
& =\tau^{T} \dot{q}-F^{T} \dot{x}
\end{aligned}
$$

i.e. the sum of the total power supplied via the control and via the output port. The output force and the apparent output stiffness of the actuator are given by:

$$
F=\frac{\partial H}{\partial x}, \quad K=\frac{\partial^{2} H}{\partial x^{2}}
$$

The stiffness can be changed, without injecting any energy to the spring via the control port, by varying $q$ while satisfying

$$
\dot{q} \in \operatorname{ker} A(q, x) \quad \forall q, x
$$

Note that a guideline for the design of energy efficient variable stiffness actuators is that the apparent output stiffness is changed without changing the elongation of the springs and, therefore, the matrix $A(q, x)$ of the model should have a kernel. This means that, for such actuators, the design should decouple the output position and the output stiffness on a mechanical level.

\section{CONCEPTUAL DESIGN}

A conceptual design, which follows the guidelines of Sec. II, has been proposed in [5] and is depicted in Fig. 2. The actuator concept consists of one internal zero free length spring and two actuated degrees of freedom. More specifically, the spring is characterized by elastic constant $k$ and is connected to the output via a lever arm, whose length can be varied by a linear degree of freedom $q_{1}$, where $0<q_{1} \leq \ell$. The second internal degree of freedom $q_{2}$ determines the position $x$ of the output. It is assumed that the dimensions and working conditions are such that the angle $\alpha$ may be neglected. Due to the kinematics of the system, the state $s$ of the spring is given by $s=\ell \sin \phi$, with $\sin \phi=\frac{x-q_{2}}{q_{1}}$. The elastic energy stored in the spring is given by the energy function $H(s)=\frac{1}{2} k s^{2}$. Then, the force

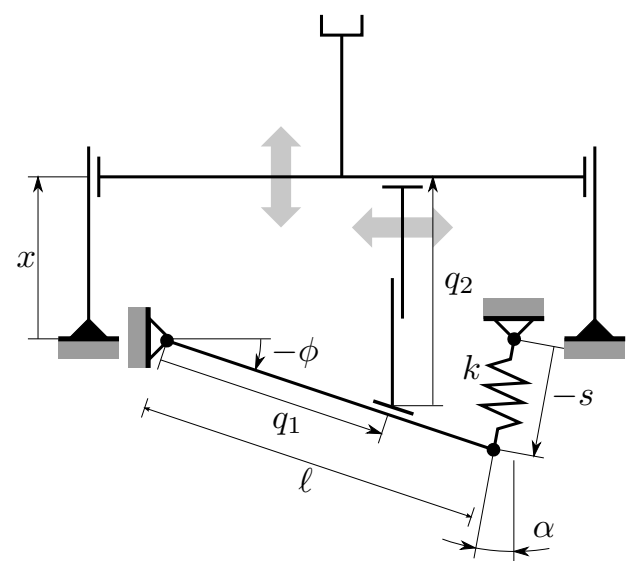

Fig. 2. Conceptual design of an energy efficient variable stiffness actuator The design is based on a lever arm with variable effective length, determined by the linear degree of freedom $q_{1}$, with $0<q_{1} \leq \ell$. The linear degree of freedom $q_{2}$ controls the equilibrium of the output position $x$. The stiffness at the output only depends on $q_{1}$ and on the elastic constant $k$ of the linear zero free length spring, whose state is $s$.

and the stiffness felt at the output port are respectively given by

$$
F=\frac{\partial H}{\partial x}=k\left(\frac{\ell}{q_{1}}\right)^{2}\left(x-q_{2}\right), \quad K=\frac{\partial^{2} H}{\partial x^{2}}=k\left(\frac{\ell}{q_{1}}\right)^{2}
$$

For this concept, in Eq. (1) we have

$$
\begin{aligned}
& A(q, x):=\left[A_{1}(q, x) \quad A_{2}(q, x)\right]=-\frac{\ell}{q_{1}}\left[\begin{array}{ll}
\sin \phi & 1
\end{array}\right] \\
& B(q):=\frac{\ell}{q_{1}}
\end{aligned}
$$

Note that, accordingly to the design guidelines, the matrix $A(q, x)$ has a kernel and, therefore, the output stiffness can be changed without supplying energy via the control port.

\section{THE VARIABLE STIFFNESS ACTUATOR PROTOTYPE}

\section{A. Realization}

The conceptual design described in Sec. III has been realized in a test setup, shown in Fig. 3, in order to provide a proof of concept. The realization closely matches the concept, with the exception that the zero free length linear spring has been replaced by an antagonistic spring setup acting on the rotation axis of the lever arm. The springs appear as a rotational spring with an elastic constant of $k=0.68 \mathrm{Nm} / \mathrm{rad}$. This arrangement is easier to realize, while it results in exactly the same system behavior. As a result, there is no longer a need to consider a specific working condition for which $\alpha$ can be neglected. Moreover, the length $\ell$ of the lever arm no longer appears in the kinematics.

The two linear internal degrees of freedom are actuated by spindle drives with Maxon A-max brushed DC motors [7]. Sliders provide the kinematic constraints depicted in Fig. 2. 


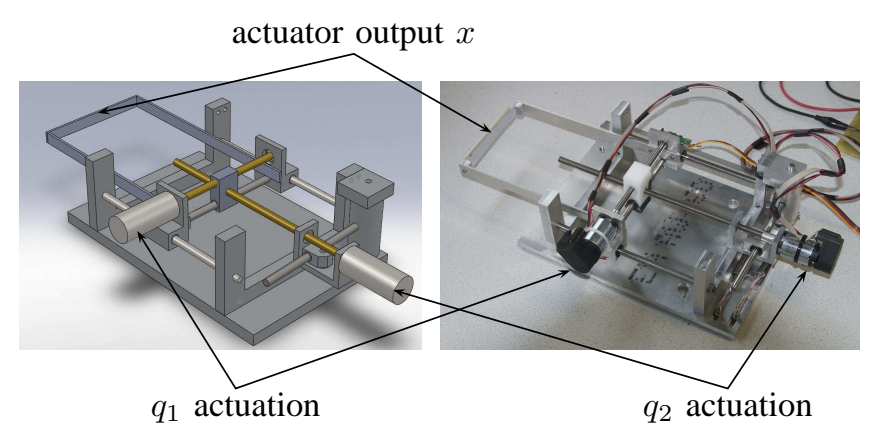

Fig. 3. Prototype design and realization - The design closely matches the concept presented in Fig. 2. The main difference is in the implementation of the zero free length spring, which is realized by using an antagonistic spring setup acting on the rotation point of the lever arm.

\section{B. Model}

Before proceeding with the experimental tests for the validation of the concept, we build the model of the setup in order to verify if the real data are comparable with the simulation results. In particular, by detailing the Dirac structure of Fig. 1, we derive the bond graph model of Fig. 4, which represents both the conceptual design and the real system since there is no substantial difference between them.

The multidimensional port $(\tau, \dot{q})$ of Fig. 1 is split into two separate control inputs, which separately actuate the internal degrees of freedom $q_{1}$ and $q_{2}$. The subsystems labeled by $M_{1}$ and $M_{2}$ contain all the relevant dynamical properties of the two motors, as specified by the data sheets, and the spindle drives.

The two MTF-elements (modulated transformers) implement the matrix $A(q, x)$ of the Dirac structure (1), as given in Eq. (6). The 0-junction represents a shared effort (the torque) on the connected bonds and a summation of the flows (velocities). The third $\mathbb{M T} \mathbb{F}$-element implements the matrix $B(q)$ of the Dirac structure (1), as given in Eq. (6). The 1-junction represents a shared flow on the connected bonds and represents the actuator output port with velocity $\dot{x}$ and force $F$. Moreover, in this model, we consider that the system is actuating a load with inertial and friction properties modeled by the $\mathbb{I}$-element and the $\mathbb{R}$-element, respectively. In the experiments, we consider a load with mass $m=0.06 \mathrm{~kg}$ and a friction coefficient $r=20 \mathrm{Ns} / \mathrm{m}$. The value of the friction coefficient is due to the high friction in the sliders supporting the output motion and has been experimentally estimated.

\section{SIMULATION AND EXPERIMENTAL RESULTS}

In this Section, we present a comparison between the simulation results of the model presented in Sec. IV-B and the experimental data, both obtained in two different scenarios. In order to have commensurable data, we implement in both simulation and real setup the same controllers, namely PID controllers on the velocities $q_{1}$ and $q_{2}$, with properly tuned parameters. Using the 20-sim simulation package [8], and its $4 \mathrm{C}$ toolchain, it is possible to simulate the bond graph model of Fig. 4 and directly export the controllers used in

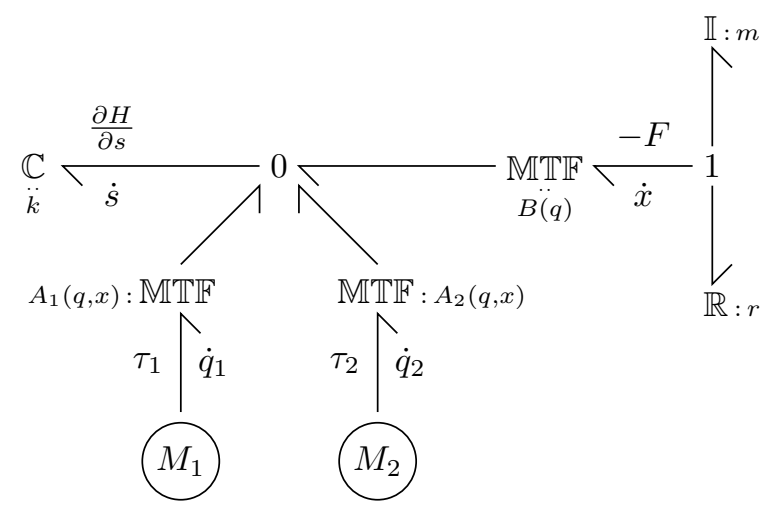

Fig. 4. Bond graph based model of the prototype design - The $\mathbb{M T F}$ elements model the Dirac structure as given in Eq. (6). The subsystems $M_{1}$ and $M_{2}$ model the actuation of the degrees of freedom $q_{1}$ and $q_{2}$ respectively. The $\mathbb{I}$-element and the $\mathbb{R}$-element model, respectively, the inertial and friction properties of the actuated load.

this simulation to $\mathrm{C}$ code, which can then run on an external controller board to actuate the real setup.

Experiment 1 - Static output force: In the first experiment, the aim is to determine a relation between the output force $F$ and the configuration $q_{1}$, which is directly related to the output stiffness by Eq. (3). The force is measured while the output position $x$ is fixed (i.e. $\dot{x}=0$ ). The claim is that the stiffness can be changed without changing the energy stored in the spring. To achieve this, the spring is loaded and the degrees of freedom are actuated while satisfying Eq. (4).

The experiment is summarized in Fig 5. The output position $x$ is fixed, $q_{1}$ is set at a distance of $0.076 \mathrm{~m}$ to the rotation point of the lever arm, $q_{2}$ is such that the angle $\phi=0.15 \mathrm{rad}$. Then, $q_{1}$ is moved towards the rotation point in $0.005 \mathrm{~m}$ increments towards the final value of $0.026 \mathrm{~m}$, while $q_{2}$ is actuated according to Eq. (4). This implies that $\phi$ and $\sin \phi=\frac{x-q_{2}}{q_{1}}$ are kept constant. After each increment, the output force in Eq. (5) is measured, and it is expected that it varies such that $F\left(q_{1}\right)=\gamma q_{1}^{-1}$, where $\gamma=k \sin \phi=0.101$ is obtained from the kinematic analysis of the design.

The results for this experiment are presented in Fig. 6, in which the averages of the measured output force for a number of values of $q_{1}$ are shown, together with the standard deviation $\sigma$. The mean values of the experiments are, except for $q_{1}=0.076 \mathrm{~m}$, all within $1 \sigma$ of the theoretic curve. The deviation for $q_{1}=0.076 \mathrm{~m}$ can be explained by stiction present in the system. For increasing values of $q_{1}$, the force generated on the output decreases by Eq. (5), but for this particular value for $q_{1}$, this force is no longer sufficient to overcome the stiction forces in the supporting sliders. If the measurements for this value of $q_{1}$ are no longer considered to be valid, the following curve can be fitted to the average values of the experiments using a least square fit, i.e. $F\left(q_{1}\right)=0.107 \cdot q_{1}^{-0.99}$, with a residual $r^{2}=0.97$. These results show that, in the prototype, the output stiffness can be changed in an energy free way, i.e. while the energy stored in the springs is not changed.

Experiment 2 - Dynamic output displacement: In the 

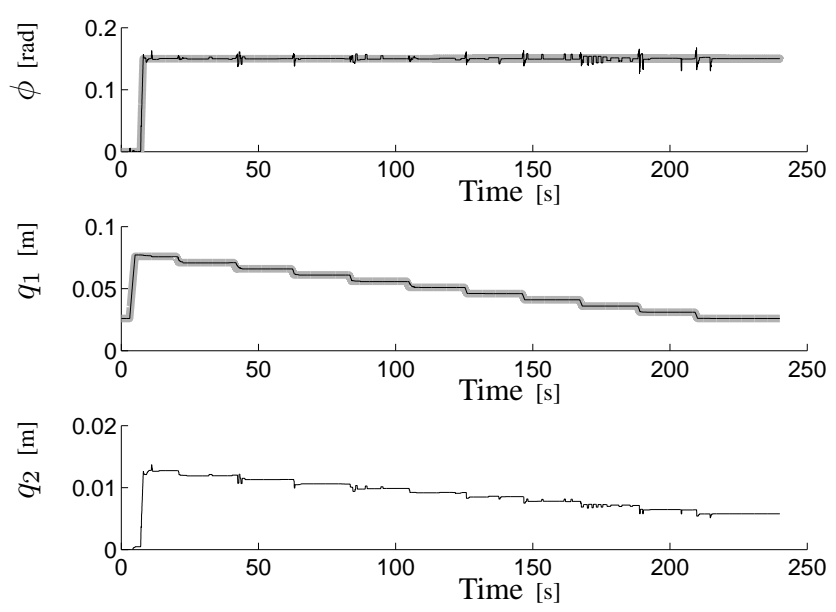

Fig. 5. Static output force measurements - While the spring is loaded, $q_{1}$ is varied along its configuration range. $q_{2}$ is varied satisfying Eq. (4), so that $\phi$ is kept constant and no energy is injected into or removed from the spring. The grey lines represent the set point values for $\phi$ and $q_{1}$, while the solid black lines represent the measured experimental values.

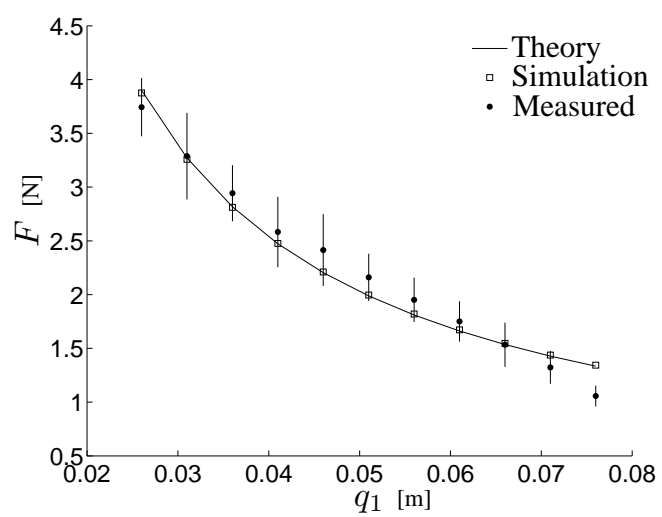

Fig. 6. Theoretic and measured output force - The solid curve shows the theoretical output force. The open squares and the solid dots indicate simulation results and the average experimentally measured values respectively. The vertical bars indicate the measurement standard deviation.

second scenario, we consider that the output load consists of a mass of $0.06 \mathrm{~kg}$ and we want to displace it while keeping a constant output stiffness. With this experiment, we aim to show that, when the stiffness is kept constant, all energy supplied via the control port is used to do work on the output port. This requires that, when the mass is not accelerated, there is no energy stored in the spring. Starting from an output position $x=0$, a desired set point value $x=0.03 \mathrm{~m}$ is provided, and $q_{2}$ is actuated to achieve this output position. Since we want to have a constant stiffness, $q_{1}$ is not actuated.

The results for both simulation and experiments are presented in Fig. 7. The simulated system responds much faster to the step set point, although the settling time for both the model and the prototype is of the same order of magnitude. It can also be noted that, when $q_{2}$ is actuated to displace the load at the desired position, the spring is compressed $\phi \neq 0$ due to the inertial properties of the load and the friction in the system. However, since the spring returns to
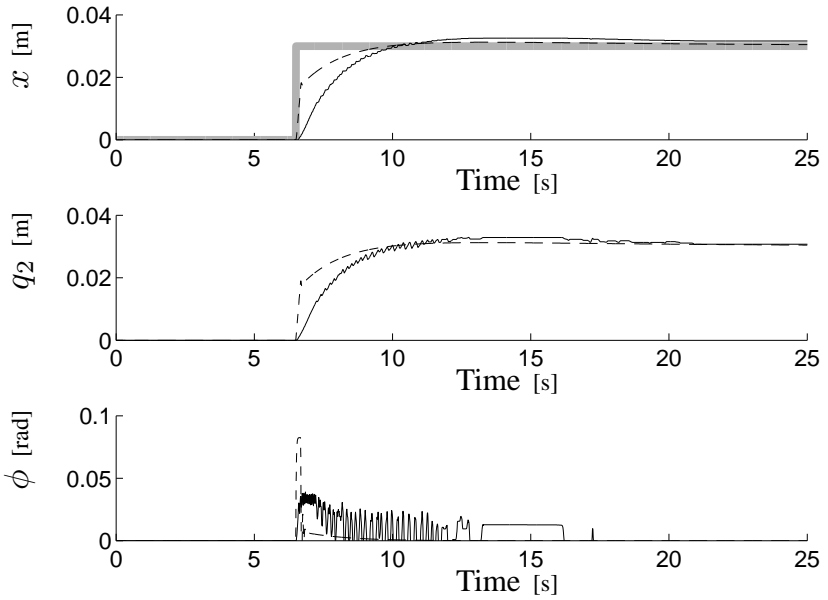

Fig. 7. Simulation (dashed) and experimental (continuous) data - The experiments consists of displacing a mass of $0.06 \mathrm{~kg}$ from $x=0$ to a desired output position $x=0.03 \mathrm{~m}$ (grey thick line). Only $q_{2}$ is actuated to achieve the desired output position. Some energy is stored in the spring when the load is accelerated, but after acceleration and reaching the set point, there is no energy left in the spring.

the uncompressed state $\phi=0$, all energy supplied via the control port is used to do work on the load. Even though the performances of the prototype can be improved by a more accurate realization, the results confirm the evaluation of the conceptual design.

\section{CONCLUSIONS AND FUTURE WORK}

In this work, a prototype realization of a novel variable stiffness actuator concept has been presented. It is shown that it is possible to realize an actuator, whose apparent output stiffness can be changed in an energy free way. This is shown in static output force measurements, in which the output force, and thus the output stiffness, is changed while keeping the energy stored in the spring constant. In dynamic experiments, it is shown that energy supplied via the control port is used only to do work at the output.

Future work will focus on designing and building new prototypes in this philosophy.

\section{REFERENCES}

[1] J.W. Hurst, J.E. Chestnutt, A.A. Rizzi, "An Actuator with Physically Variable Stiffness for Highly Dynamic Legged Locomotion", Proc. IEEE Int. Conf. on Robotics and Automation, 2004.

[2] G. Tonietti, R. Schiavi, A. Bicchi, "Design and Control of a Variable Stiffness Actuator for Safe and Fast Physical Human/Robot Interaction", Proc. IEEE Int. Conf. on Robotics and Automation, 2005.

[3] S. Wolf, G. Hirzinger, "A New Variable Stiffness Design: Matching Requirements of the Next Robot Generation", Proc. IEEE Int. Conf. on Robotics and Automation, 2008.

[4] B. Vanderborght, N.G. Tsagarakis, C. Semini, R. van Ham, D.G. Caldwell, "MACCEPA 2.0: Adjustable Compliant Actuator with Stiffening Characteristic for Energy Efficient Hopping", Proc. IEEE Int. Conf. on Robotics and Automation, 2009.

[5] L.C. Visser, R. Carloni, R. Ünal, S. Stramigioli, "Modeling and Design of Energy Efficient Variable Stiffness Actuators", Proc. IEEE Int. Conf. on Robotics and Automation, 2010.

[6] V. Duindam, A. Macchelli, S. Stramigioli, H. Bruyninckx, "Modeling and Control of Complex Physical Systems", Springer, 2009.

[7] Maxon Motor, "A-max 22 brushed DC motor", available: http://ww.maxonmotor.com, 2010.

[8] Controllab Products B.V., "20-sim", http://www.20sim.com, 2010. 